\title{
Fracturas de implantes de tibia. Consecuencias para los ensayos de los implantes
}

\author{
S. FREI, H. PLOEG, C. REINSCHMIDT, P. HEUBERGER \\ Biomechanics Department \\ Sulzer Orthopedics Ltd. \\ Winterthur, Suiza
}

\section{Resumen}

El objetivo de este trabajo es estudiar los modelos de fracturas de prótesis de tibia descritos en la literatura $y$, sobre la base de dichos conocimientos, desarrollar un ensayo para implantes en la cual se logren simular condiciones fisiológicas. El caso descrito con mayor frecuencia en la literatura es la migración de la parte medial de la prótesis, fenómeno que, a menudo, antecede a la fractura de la prótesis. La posición defectuosa resultante de dicha migración modifica el eje mecánico y provoca una sobrecarga en la parte medial de la prótesis. Se ha desarrollado un ensayo de implantes que simula el modelo de fallo in-vivo. Dos bases de material sintético de diferente dureza simulan la diferente densidad ósea medial y lateral, para permitir una migración del lado medial del platillo. En el ensayo dinámico el platillo tibial es sometido a una carga de un solo lado y el vástago de la prótesis se apuntala con un contrasoporte. Para simular el entorno fisiológico, todo el dispositivo se encuentra sumergido en una solución de Ringer a $37^{\circ}$. El implante de tibia es expuesto durante 10 millones de ciclos a una carga que corresponde al quíntuple del peso del cuerpo $(4.000 \mathrm{~N})$. El punto de aplicación de la fuerza fue tomado de datos de análisis del caminar (marcha en superficie plana, ascendente y descendente, subir escaleras). Con esta carga fisiológica se efectuaron los primeros ensayos. En éstas quedó demostrado que productos clínicamente exitosos podían superar el ensayo. Por el contrario, los productos con modelos de fracturas conocidos no la superaron. Los primeros resultados con estos productos demostraron también que el ensayo generaba muestras de fractura semejantes a las que se producen in vivo. Debido a la configuración fisiológica del dispositivo se somete el sistema completo es decir el platillo, el vástago de la prótesis y la unión - generalmente modular - entre ambos a ensayo. El "ensayo fisiológico de tibia " descrito ayuda a evaluar los diseños de tibia antes de que se autorice su venta. Nuevos implantes y modificaciones de diseño en implantes ya existentes son puestos a la venta después de cumplir con las exigencias del ensayo.

Palabras clave: Resistencia a fatiga. Ensayos de implantes. Prótesis. Reemplazo total de rodilla.

\begin{abstract}
Summary
The aim of this investigation was to study the failure modes of tibial implant fractures and to develop a new implant test, which simulates the physiological conditions leading to implant fractures. Typically, these fractures are proceeded by a subsidence of the tibial plateau on the medial side. This subsidence causes a varus misalignment changing the mechanical axis and resulting in an overloading of the medial side. A fatigue test has been developed which simulates the above described in-vivo failure mode. For this test, the tilting or subsidence of the tibial tray is achieved by using material with different stiffness' for the medial and lateral support. The tibial plateau is loaded exclusively on the medial side and, if applicable, the stem is loaded by a reaction force acting at distal-lateral part of the stem. To simulate the physiological environment, the implant is immersed in a Ringer solution of $37^{\circ} \mathrm{C}$. The tibial implant is fatigue-tested by applying a cyclic load of five times body weight $(4000 N)$. In order to pass this test, an implant has to withstand 10 million cycles. The load magnitude was determined from gait analyses studies (walking, stair ascending/descending). Preliminary evaluation tests showed that clinically successful implants are able to withstand these load levels for 10 million cycles whereas implants with a known history of fatigue fractures failed at these load levels. Additionally, the fracture patterns found in-vivo were similar to those produced with this test. The described ,physiological tibia test,, helps to evaluate new implant designs, before they are introduced to the market. New implants are only introduced to the market, once they have passed our fatigue tests.
\end{abstract}

Key words: Fatigue failure. Implant Testing. Prosthesis. TKR. Knee Replacement.

Correspondencia:

Susanne Frei

Biomechanics Department, Sulzer Orthopedics Ltd.

PO Box 65, CH-8404 Winterthur, Suiza. E-mail: susanne.frei@ sulzer.ch 


\section{Introducción}

Gracias a la endoprótesis los pacientes atendidos pueden retomar la vida cotidiana sin dolores. La cinemática de las articulaciones se restablece prácticamente en su totalidad, lo que permite a los pacientes llevar una vida independiente. Sin embargo, en raros casos, el proceso no se desarrolla a satisfacción del paciente. Diferentes problemas como infección, aflojamiento, luxación, dolor patelar e inestabilidad ${ }^{1,2}$ pueden ser razones que exijan una nueva intervención. Dado que sólo un porcentaje mínimo de todas las revisiones se debe a las fracturas de los implantes, éstas no suelen ser un tema tratado en congresos y jornadas aunque constituyen un problema muy grave para el paciente y que debe ser absolutamente evitado ya que, en el caso de una fractura de la prótesis, la inmediata reintervención es ineludible.

Para mantener lo más baja posible la resección ósea en la implantación de una prótesis de rodilla, se trata de fabricar platillos de tibia metálicos cada vez más finos, disminuyendo esto la resistencia del implante. Con la misma resección ósea, los platillos de metal más finos permiten el uso de insertos de PE (polietileno) más gruesos, lo que puede influenciar positivamente en la duración del $\mathrm{PE}^{17}$, ${ }^{18}$. La resistencia del implante es más o menos proporcional al cuadrado del espesor del implante. Pequeñas reducciones del espesor del platillo pueden, por lo tanto, causar una considerable pérdida de resistencia. Por un lado, está la exigencia de seguridad del implante y, por el otro, la de una pérdida ósea lo más reducida posible al realizar la implantación. Para responder a ambas exigencias, no sólo es conveniente sino, prácticamente, imprescindible estudiar el platillo tibial.

Para ello existe ya un ensayo de la American Society for Testing and Materials, ASTM $^{19}$, que fue presentada - como proposición - ante la International Organization for Standarization (ISO). En este ensayo, llamada "cantilever bending test", se fija de un solo lado la mitad del platillo y el lado opuesto se somete a una carga en aumento cuyo valor normalmente no está determinado. Sin embargo, debido al tipo de configuración de este ensayo, la fuerza no puede corresponder a la carga fisiológica. Nuestras experiencias con el "cantilever bending test" lo han demostrado. Con este tipo de ensayo, productos de demostrada eficacia clínica no han logrado soportar cargas fisiológicas. Además, nuestras experiencias han demostrado que el "cantilever bending test" sólo reproduce uno de los distintos tipos de fracturas in vivo. La estructura del "cantilever bending test" no es fisiológica. Tampoco ensaya el sistema completo ya que la influencia de un vástago de anclaje y, si lo hay, de una unión modular entre platillo y vástago, no son tomadas en cuenta.

Por tal motivo, el objetivo del presente estudio es desarrollar un nuevo tipo de ensayo que considere las condiciones fisiológicas y reproduzca los modelos de fractura in vivo. Para alcanzar este objetivo se elaboraron primero las bases para los modelos de fracturas de prótesis de tibia.

\section{Bases}

\section{Modelos de fracturas de prótesis de tibia}

En la literatura el problema que se describe con mayor frecuencia es la migración del lado medial de la prótesis que, a menudo, antecede a la fractura del implante. En esos casos, o bien el implante fue colocado con una posición defectuosa, de orientación varus, o la migración mencionada fue la que provocó este defecto ${ }^{1,3,6}$. No es sorprendente que el platillo medial se hunda si no se cuenta con un apoyo óseo suficiente ya que - aún en el caso de un alineamiento correcto - la carga sobre el cóndilo medial siempre es mayor que sobre el lateral ${ }^{20,21}$. La posición defectuosa del implante modifica el eje mecánico y de esto resulta una sobrecarga cada vez mayor en el lado medial de la prótesis.

Característico en estos casos es el frecuente aflojamiento de los componentes de la tibia que antecede a la fractura de la prótesis. Sin embargo, no siempre está claro si lo primero es la rotura de la prótesis de tibia y, debido a ello, se produce la migración, o viceversa.

Varias son las razones que pueden originar una fractura de la prótesis. Generalmente la fractura es el resultado de varios factores. Las causas de las fracturas pueden dividirse a grosso modo en cinco categorías: (a) calidad ósea, (b) carga, (c) fijación, (d) técnica quirúrgica y (e) diseño del implante.

(a) Calidad ósea:

La causa de las fracturas de los implantes de tibia es, a menudo, la calidad deficiente de los huesos. Esta última puede estar disminuida ya antes de la operación o empeorar en la etapa postoperatoria. En el postoperatorio la calidad ósea puede reducirse como consecuencia de infecciones, reacciones a cuerpos extraños o "stress shielding". Otras razones de una mala calidad ósea pueden ser 
también $\operatorname{artritis}^{2} \mathrm{u}$ osteoporosis avanzadas. Si el lecho óseo es insuficiente ya antes de la operación ${ }^{5}$, el resultado puede ser el mismo: migración unilateral de la prótesis, defecto de posición, sobrecarga y, en el peor de los casos, fractura del implante. Aún cuando la fijación del anclaje de la prótesis en el canal intramedular sea buena, un implante puede romperse si el apoyo óseo o el cemento no son suficientes ${ }^{3,8}$.

\section{(b) Carga:}

Al hablar de carga, el peso y la actividad ${ }^{3-5}$ del paciente son factores determinantes y éste último puede influenciar directamente. Exceso de peso y demasiada actividad hacen que las articulaciones estén sobrecargadas. Esto provoca una sobrecarga de la prótesis y del hueso, la cual puede causar la fractura del implante. Sin embargo, siempre es necesario un mínimo de carga para poder conservar la calidad de los huesos ${ }^{22}$.

\section{(c) Fijación:}

Una buena fijación del platillo tibial es un factor determinante para una larga vida, tanto para prótesis cementada como para aquéllas fijadas sin cemento. Un aflojamiento en las prótesis cementadas o una fijación insuficiente del platillo en las prótesis sin cemento ${ }^{7,9-11,13}$ pueden ser responsables también de una fractura del implante.

(d) Técnica quirúrgica:

La técnica quirúrgica puede ser determinante para la duración de un implante. Es de fundamental importancia el posicionamiento de la prótesis durante la operación. Si el defecto óseo es grande, se puede llenar con cemento para huesos. Al enfriarse, el cemento se contrae y, debido a ello, se forma una hendidura entre el cemento y el hueso. $\mathrm{O}$ sea que, en esa zona, la prótesis está floja. $\mathrm{Al}$ ser sometido a cargas, hay movimiento y el implante se puede romper ${ }^{7}$. Un mal o insuficiente alineamiento del platillo tibial en la intervención también puede favorecer la fractura del implante ${ }^{14,16}$. El platillo no sólo debe cubrir la mayor superficie posible del hueso, sino quedar apoyado sobre el hueso cortical $^{23}$. Otro factor que puede favorecer la fractura del implante es una posición defectuosa del hueso, con orientación varus ${ }^{2}$, anterior a la operación.

(e) Diseño del implante:

Finalmente, también el implante influye en los resultados a largo plazo. En la literatura varios diseños de implantes fueron considerados, en parte, insuficientes ${ }^{1,} 7-9,16$. Son determinantes para la resistencia de un implante no sólo el espesor del mismo, sino también los orificios ranuras. $\mathrm{Si}$ existen orificios en una zona muy solicitada, se reduce considerablemente la resistencia ${ }^{8}$. Con respecto a los materiales empleados, la técnica de elaboración de los mismos desempeña un papel determinante. La resistencia a la fatiga se puede reducir al colocar una macroestructura adicional. En este tipo de procesos se agregan ulteriormente estructuras al implante, del lado del hueso, las cuales, en los implantes sin cemento, están destinadas a favorecer la adherencia del implante al hueso. Para ello aveces se elige aplicar también un procedimiento de sinterización en el cual el material de base es calentado. Este calentamiento provoca un crecimiento de los gránulos del material de base lo que, a su vez, reduce la resistencia a la fatiga. Debido a ello el implante puede debilitarse de tal forma que ya no es capaz de responder a las exigencias $^{4,9}$. El tipo de superficie también influye en la resistencia a la fatiga del platillo tibial. Superficies lisas y finamente granuladas tienen mejores propiedades mecánicas a largo plazo que las estructuradas o de gránulos más gruesos. Distintos tipos de elaboración mecánica como, por ej. el perdigonado fino, pueden producir esfuerzos internos en el material, aumentado la resistencia perimetral a la flexión.

El mecanismo de fractura ${ }^{1-7,11,23}$ se representa esquemáticamente en la Figura 1. La Figura 1a muestra una buena situación postoperatoria. Incluso en una rodilla normal, el lado medial siempre presenta mayor solicitud de cargas $^{20,21}$. Si en el lado medial el apoyo óseo es insuficiente, si la carga es demasiado grande, si el implante no está correcta y debidamente fijado o si las fuerzas son distribuidas de modo insuficiente por el platillo tibial, el resultado puede ser un defecto de posición, con orientación varus (Figura. 1b). Como consecuencia de esto también puede hundirse el lado medial del platillo y el lado lateral puede, incluso, levantarse (Figura. 1c). Si hay un vástago de fijación, éste puede permanecer bien fijado o estar en contacto con la sustancia cortical del canal intramedular.

\section{Bases de los ensayos de implantes}

En los componentes metálicos de los implantes de tibia, la fractura in vivo se produce, generalmente, debido a la fatiga. La resistencia a la fatiga es una propiedad mecánica de los materiales e indica que tensiones máximas puede resistir un material ante una carga oscilante durante un tiempo prolongado. En el caso de una carga que se repite, la resistencia es inferior a la que se constata si el material es expuesto a una única carga. Este comportamiento se representa en la Figura 2, en el 


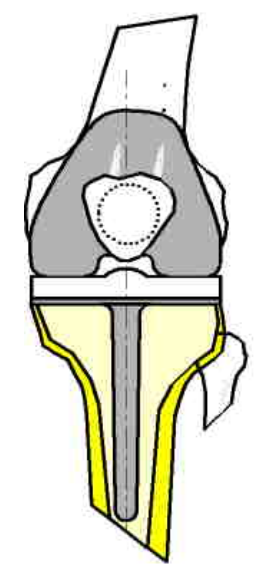

a

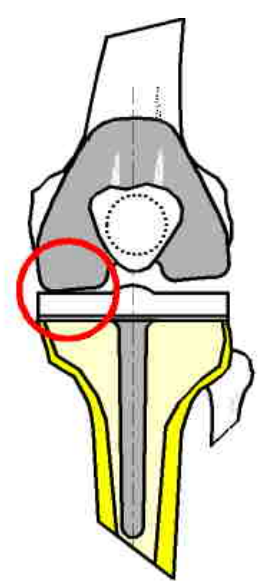

b

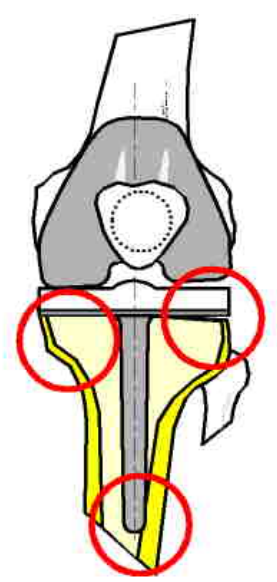

c

Figura 1: Representación esquemática de un proceso de fractura in vivo. a) Buen alineamiento postoperatorio. b) Posición defectuosa varus con carga medial. c) Migración medial y levantamiento lateral de la prótesis, el vástago se encuentra en contacto con la cortical.

llamado diagrama de tensiones-ciclos (diagrama S$N)$. La amplitud de esfuerzo $\sigma_{a}$ es trazada con respecto al número de ciclos $\mathrm{N}$. $\sigma_{\mathrm{u}}$ corresponde a la resistencia a la tracción y $\sigma_{\mathrm{f}}$ al periodo de vida. Las prótesis de tibia deben ser estudiadas siempre en relación con su periodo de vida ya que, al caminar, el paciente somete a carga constantemente al implante. Se parte del hecho que un paciente sano y activo realiza entre 1 y 2 millones de pasos por año ${ }^{24}$.

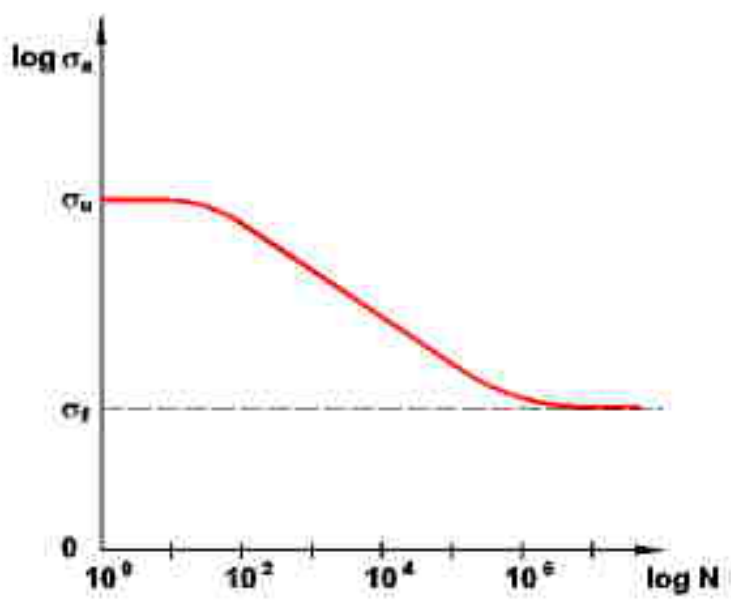

Figura 2: Diagrama de ciclos de esfuerzo. La curva representa esquemáticamente la resistencia en relación con el número de ciclos.

La duración de un implante depende de diferentes factores. Los más importantes son: el material, la geometría, las características de las superficies, la forma de los orificios, la temperatura y el medio de trabajo. Estos factores, en parte, pueden ser simulados muy difícilmente - o no pueden ser simulados - en modelos de elementos finitos por ordenador. Por tal motivo, aun hoy, el control de los componentes, sobre todo en relación con su resistencia a la fatiga, sigue siendo una parte determinante del control de calidad de los fabricantes de prótesis.

\section{Método de ensayo}

En la Figura 3 se representa el ensayo denominado a continuación "ensayo fisiológico de tibia" y que simula las fracturas de implantes arriba descritas. El platillo tibial se apoya sobre dos bases de material sintético que simulan el hueso esponjoso. Las bases son de diferentes materiales. El material de la base lateral es un elastómero de uretano muy resistente a la presión y capaz de soportar grandes cargas. El material de la base mediall es un poliuretano espumado. Esta de material sintético más blanda permite simular una migración del lado medial del platillo. Este último sólo es sometido a carga en su parte medial. La carga corresponde aproximadamente al quíntuple del peso del cuerpo $(4.000 \mathrm{~N})$.

Si el implante tiene un vástago de anclaje, éste se apuntala en el extremo distal con un contrasoporte que simula el apoyo del vástago de la tibia contra el hueso cortical del canal intramedular. El extremo distal del vástago se fija con cemento para huesos en el interior de un casquillo, en cuya cara lateral se coloca una esfera apoyada sobre un espaciador de polietileno. Este último se coloca entre la pared del dispositivo y la esfera. Ya que el 

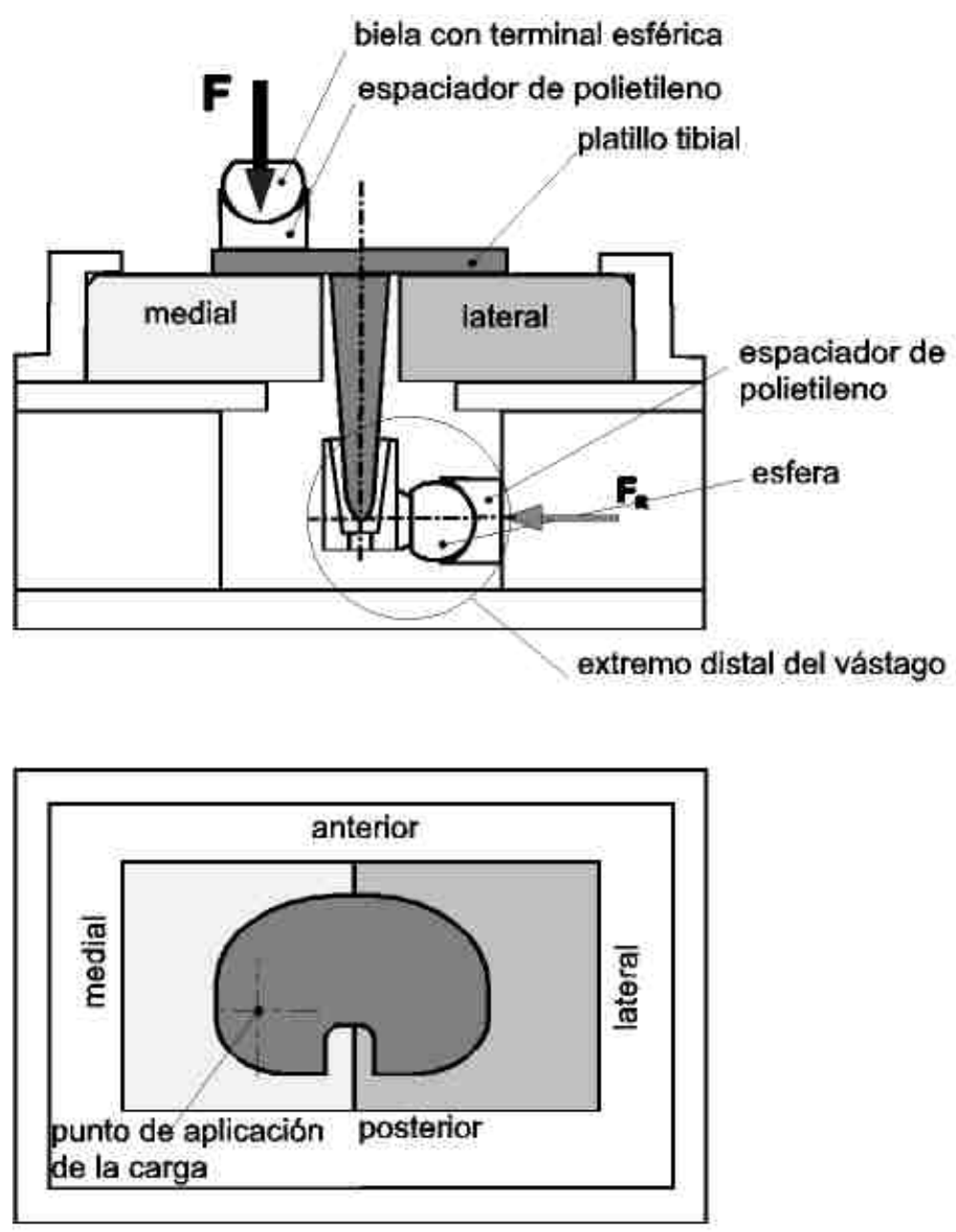

Figura. 3: Esquema del ensayo fisiológico de tibia (vista frontal y proximal).

espaciador de polietileno puede deslizarse libremente en la pared del dispositivo, es posible un movimiento del extremo distal del vástago en el plano sagital. Se evita un movimiento de traslación del extremo distal del vástago en el eje mediolateral. Si el platillo tibial es sometido a una carga medial, la base blanda de material sintético cede. Se produce la migración medial del platillo tibial y el extremo distal del vástago es apretado contra la pared del dispositivo por el contrasoporte. Se genera así la fuerza de reacción $\mathrm{F}_{\mathrm{R}}$. La fuerza de reacción provoca una carga de flexión del vástago. En éste último el momento es más fuerte en la zona de unión al platillo. En sistemas modulares la unión entre el vástago y el platillo es una zona mecánicamente crítica. Con este tipo de ensayo se somete precisamente esta región a la mayor carga.

La carga es aplicada a través de una biela con terminal esférico. Un espaciador de polietileno protege al platillo contra una carga puntual demasiado elevada. El implante se somete a carga por 10 millones de ciclos por dos razones: 10 millones de ciclos corresponden aprox. a 5-10 años de duración del implante. Más importante en relación con esto es, sin embargo, que la probabilidad de una fractura después de 10 millones de ciclos es muy baja. Esto significa que si hasta entonces no se produjo ninguna fractura, debido a la forma de curva asintótica del diagrama $\mathrm{S}-\mathrm{N}$, se puede partir del hecho que la tensión ejercida es más pequeña que la resistencia a la fatiga del material (Figura 2). Si hasta esos 10 millones de ciclos no hay ninguna fractura, se aumenta la carga entre un 30 y $50 \%$ y se vuelve a probar la prótesis 10 millones de ciclos. El ensayo se considera terminado cuando se rompe el implante. La fractura puede estar en el platillo o en el vástago. Si la fractura se produce con la carga básica, el implante no superó el ensayo. Si se produce después de incrementar la carga, el 
implante pasó el ensayo. Al aumentar la carga, el valor de la carga de fractura provee información sobre el factor de seguridad. Si el factor de seguridad es muy grande, se puede, dado el caso, considerar una reducción del espesor del implante para mantener la resección ósea lo más baja posible. El punto débil del implante se determina probando el platillo tibial hasta su fractura.

La carga es de forma sinuosoidal y oscilante. La relación entre baja y alta carga es de 0.1 , lo que corresponde a la relación entre esfuerzo máximo y mínimo habitualmente aplicada en los controles de prótesis. La frecuencia de ensayo influye en los resultados del ensayo en la medida en que a altas frecuencias materiales sintéticos se vuelven más rígidos, no pudiendo acompañar la deformación o calentamiento lo que, a su vez, modifica las propiedades del material. Para tomar en cuenta los efectos de la corrosión, el control se hace en una solución de Ringer ( $0.9 \%$ solución salina) a una temperatura de $37^{\circ} \mathrm{C}$.

\section{Resultados y discusión}

En la Figura 4a se resumen esquemáticamente líneas de fracturas de tres publicaciones diferentes ${ }^{1}$, 3, ${ }^{4}$. En todos los casos se trata de diseños con conservación del ligamento cruzado. La fractura del platillo posterior estabilizado de Abernethy (1996) no se cuenta ya que no representa problemas para el ligamento cruzado posterior y, por lo tanto, posee una forma básica diferente. Las fracturas se produjeron siempre en el lado medial del platillo. Estas tres publicaciones consideran en total 19 platillos rotos. Las fracturas se encontraban en una línea medio-lateral o en una combinación de una fractura medio-lateral con una fractura adicional de recorrido antero-posterior.

En la Figura 4b se representa esquemáticamente un platillo tibial sometido al ensayo fisiológico de tibia. La línea de fractura corresponde aquí a una línea medio-lateral sobre el lado del platillo exigido. La superficie de fractura es parecida a la mencionada en la literatura. Con el mismo platillo también se efectuó un "cantilever bending test"19. El platillo estudiado se puede ver en la Figura 4c. La línea de fractura tiene un desarrollo puramente antero-posterior. Debido a la configuración del "cantilever bending test", siempre se genera este tipo de fractura. El "cantilever bending test" ha sido desarrollado para hacer comparaciones de la resistencia a la fatiga de diferentes platillos tibiales metálicos bajo condiciones de ensayo semejantes. El ensayo fisiológico es más complicado y, por lo tanto, más difícil de representar pero está mejor adaptado a la fisiología y se pueden reproducir fracturas in vivo vistas en explantes.

En nuevos productos o en el caso de modificaciones importantes de productos existentes, en Sulzer Orthopedics se llevan a cabo ambos ensayos y la venta de los implantes sólo se autoriza cuando éstos logran superar ambos ensayos. Con el "cantilever bending test" se cubre, generalmente, la norma vigente. Además, el ensayo permite obtener buenos resultados de comparación. Con el ensayo fisiológico de tibia se prueba el implante con una carga fisiológica y en una configuración fisiológica.
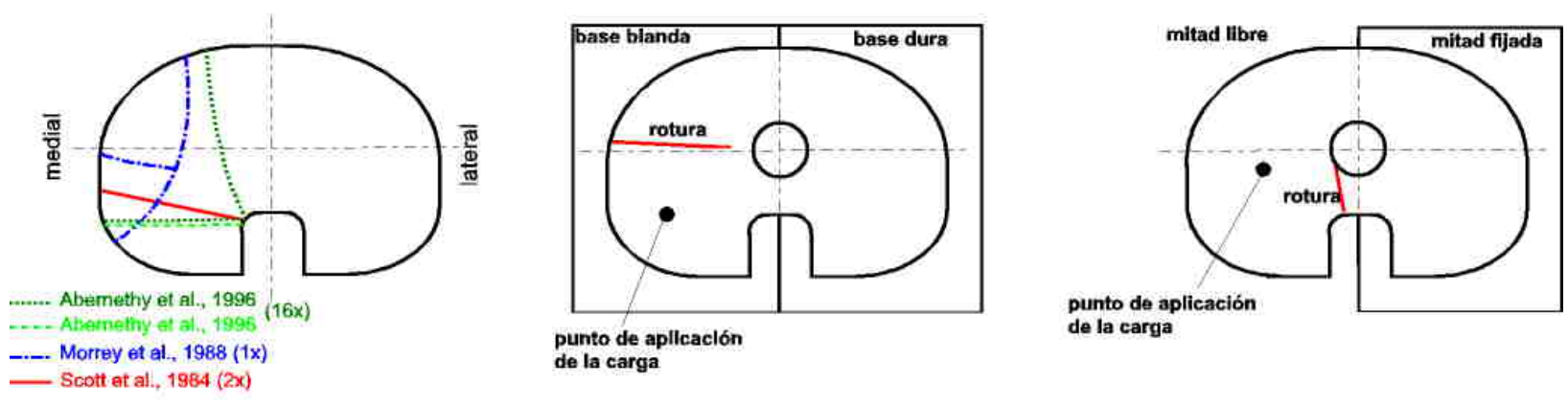

Figura 4: a) Resumen de líneas de fractura descritas en la literatura. Esquema de un implante probado con b) la prueba fisiológica y c) el "cantilever bending test". 


\section{Conclusiones}

El "ensayo fisiológico de tibia" es una ayuda para probar prótesis de tibia nuevas o modificadas. Se pueden localizar y, dado el caso, eliminar las deficiencias de diseño, materiales y técnicas de fabricación. Gracias al ensayo combinado de platillo, vástago y la unión entre ambos en una misma prueba, se logra un ahorro de tiempo y dinero.

Pruebas individuales no ofrecen información acerca del sistema en su totalidad. La tendencia actual en el desarrollo de prótesis de rodilla se dirige hacia el desarrollo de sistemas modulares. Por tal razón, el "ensayo fisiológico de tibia" gana en importancia.

La investigación de la literatura sobre fracturas in vivo ha demostrado que las fracturas de las prótesis de tibia siguen un determinado esquema. Mediante modelos de ordenador adecuados se pueden optimizar las prótesis y, a continuación, a través de controles específicos, probarlas en laboratorio para que soporten también condiciones extremas in vivo y, de este modo, respondan a las exigencias fisiológicas actuales.

\section{Bibliografía}

1. Abernethy PJ, Robinson CM, Fowler RM. Fracture of the metal tibial tray after Kinematic total knee replacement. A common cause of early aseptic failure. J Bone Joint Surg Br 1996;78:2205.

2. Insall JN, Dethmers DA. Revision of total knee arthroplasty. Clin Orthop 1982;123-30.

3. Scott RD, Ewald FC, Walker PS. Fracture of the metallic tibial tray following total knee replacement. Report of two cases. J Bone Joint Surg Am 1984;66:780-2.

4. Morrey BF, Chao EY. Fracture of the porouscoated metal tray of a biologically fixed knee prosthesis. Report of a case. Clin Orthop 1988;1829.

5. Gradisar IA, Jr., Hoffmann ML, Askew MJ. Fracture of a fenestrated metal backing of a tibial knee component. A case report. J Arthroplasty 1989;4:27-30.

6. Lotke PA, Ecker ML. Influence of positioning of prosthesis in total knee replacement. J Bone Joint Surg Am 1977;59:77-9.

7. Mendes DG, Brandon D, Galor L, Roffman M. Breakage of the metal tray in total knee replacement. Orthopedics 1984;7:860-2.

8. Flivik G, Ljung P, Rydholm U. Fracture of the tibial tray of the PCA knee. A case report of early failure caused by improper design. Acta Orthop Scand 1990;61:26-8.

9. Ranawat CS, Johanson NA, Rimnac CM, Wright TM, Schwartz RE. Retrieval analysis of porous-coated components for total knee arthroplasty. A report of two cases. Clin Orthop 1986;244-8.

10. Tjörnstrand B, Lidgren $\mathbf{L}$. Fracture of three cases of tibial component failure. Acta Orthop Scand 1985;56:127-9.

11. Jansson V. [Mechanical loading and biomechanical problems of total joint surface replacement of the tibial plateau] Mechanische Belastung und biomechanische Probleme des kompletten Gelenkflachenersatzes beim Tibiaplateau. Z Orthop Ihre Grenzgeb 1990;128:606-11.

12. Bryan RS, Rand JA. Revision total knee arthroplasty. Clin Orthop 1982;116-22.

13. Goldberg VM. Controversies of Total Knee Arthroplasty. Raven Press, New York, 1991;51-60

14. Shoji H, D'Ambrosia RD, Lipscomb PR. Failed polycentric total knee prostheses. J Bone Joint Surg Am 1976;58:773-7.

15. Dannenmaier WC, Haynes DW, Nelson CL. Granulomatous reaction and cystic bony destruction associated with high wear rate in a total knee prosthesis. Clin Orthop 1985;224-30.

16. Rand JA, Bryan RS. Revision after total knee arthroplasty. Orthop Clin North Am 1982;13:20112.

17. Bartel DL, Burstein AH, Toda MD and Edwards DL. The effects of conformity and plastic thickness on contact stresses in metal-backed plastic implants. J.Biomech.Eng. 107:193-199, 1985.

18. Bartel DL, Bicknell VL and Wright TM. The effect of conformity, thickness and material stresses in UHMWPE components for total joint replacement. J.Bone Joint Surg.Am. 68-A:10411051, 1986.

19. ASTM. Standard Test Method for Cyclic Fatigue Testing of Metal Tray Components of Total Knee Joint Replacement. F1800-97

20. Morrison JB. The mechanics of the knee joint in relation to normal walking. J Biomech 1970;3:5161.

21. Harrington IJ. Static and dynamic loading patterns in knee joints with deformities. J Bone Joint Surg Am 1983;65:247-59.

22. Cowin SC. Bone mechanics. CRC Press, Inc, Boca Raton, 1989. Chapter 10

23. Cameron HU, McNeice GM. Mechanical failure modes in total knee replacement. Arch Orthop Trauma Surg 1981;98:135-8.

24. Styles CM, Gregson PJ. Development of fatigue lifetime predictive test methods for THRs. AM1 Report.Anonymous AEA Technology. , 1996 